\title{
Is there an appropriate time to initiate the rehabilitation of human gametes destined for human ARTs?
}

\author{
David F. Albertini
}

Published online: 10 March 2015

(C) Springer Science+Business Media New York 2015

It is an unavoidable tenet in the practice of human ARTs that at some stage or another, gametes will be collected and united ex vivo with the expressed purpose of generating embryos. Whether those embryos will carry with them the toolkit that endows them with the ability to attach to the endometrium, effect implantation, and propagate a maternal environment conducive to the support of a term gestation is another matter altogether. In fact, few amongst us would doubt the importance of the embryonic toolkit nor would doubts be raised that the toolkit itself is comprised of assorted devices that arose during the differentiation and maturation of eggs and sperm. Think mitochondria, centrioles, chromosomes, gap junctions, and ribosomes to name a few.

The protracted and sometimes convoluted course of gametogenesis is by necessity subject to the prevailing conditions of the microenvironment within which our gametes residenamely, follicles and seminiferous tubules. Gonads from the very beginning establish the gender-specific niche that determines which of our prospective gametes will make the grade and assume the card-carrying identity of a developmentally competent sperm or egg. It follows then that the cumulative wrath of aging, environmental exposures, and lifestyle habits (good or bad) in our gonads sets the stage for the arrival of what are likely to be compromised gametes from which the ART clinic is being asked to generate good embryos. Is it

Capsule While turning the clock back on our pristine germ cells is a lofty endeavor embraced by the regenerative medicine community, the more tangible strategy of recognizing and dealing with gamete deficits intrinsic to natural aging, lifestyle habits, or acquired as a result of ARTs is a worthwhile future goal for the treatment of infertility.

D. F. Albertini $(\bowtie)$

University of Kansas Medical Center, Kansas City, KS, USA

e-mail: dalbertini@kumc.edu possible to rehabilitate gametes before or after their appearance in the embryology laboratory?

Gametes procured for infertility treatments are accorded all of the environmentally favorable conditions as best as can be recapitulated in the embryology laboratory. While some strategies for gamete rehabilitation remain within the purview of the "experimental"-in vitro maturation of oocytes, cytoplasmic/mitochondrial transfer—others have gained notoriety as short-term solutions just prior to or during fertilization. In this issue, an example of this acute form of rehab is illustrated in the paper by Rubino and colleagues in which a sibling oocyte comparison is reported querying the potential benefits of myoinositol supplementation during semen preparation for ICSI (see Improving fertilization rate in ICSI cycles by adding myoinositol to the semen preparation procedures: a prospective, bicentric, randomized trial on sibling oocytes, 10.1007/s10815-014-0401-2). "Curing what ails you" through the use of medium additives or nanosurgical interventions will continue to be an important arena for development of methods and approaches with which to effect rehabilitation of gametes, at least from the perspective of sperm or egg. But the more long-range strategy for planning ahead and correcting problems before gamete retrieval has entered into and become confluent with the notion that better health means better quality gametes.

The importance of pre- or peri-conceptional health is certainly not new, and one need only reflect momentarily on the impact folic acid supplementation has had on reproductive health outcomes. However, as more and more evidence points to the potential value of vitamin supplementation or screening for patients planning ART, the burden of proof regarding costs and benefit remains a matter of dispute. Fortunately, and as reported in JARG this month, some groups have taken this issue to heart, and for the case of vitamin $\mathrm{D}$, we encourage you 
to examine the paper by Pacis and associates who conclude that wide-scale screening and supplementation are not justified at this time for patients anticipating infertility treatment (Vitamin D and assisted reproduction: should vitamin D be routinely screened and repleted prior to ART? A systematic review 10.1007/s10815-014-0407-9). Yet another example of preconceptional treatments for ART patients, and one gaining in utilization internationally (but not necessarily reporting-see below) is that of metformin. Christianson and colleagues provide the JARG readership this month with a world-wide perspective of metformin use in infertility clinics in a web-based study indicating both wide variability in adoption between clinics and an overall vote of non-confidence with respect to therapeutic value.

While trends in improving general health to optimize fertility chances will continue to be proposed through the nutraceutical or pharmaceutical channels, the conundrum posed by the everexpanding obesity (pun intended) epidemic and infertility is raising more questions than ever before. That a genetic linkage contributes to obesity and infertility has long been proposed, and next month JARG will cover this topic in review form (see Butler et al., JARG DOI 10.1007/s10815-014-0411-0). More timely and thought provoking, however, is a recent publication consonant with the theme of gamete rehabilitation in which the impact of obesity on oocyte mitochondria has been shown to underscore impaired offspring health in what may be a therapeutically accessible treatment modality.

Rebecca Robker and her Australian collaborators have taken a major step forward towards understanding exactly what it is at a molecular-cellular level in obese mothers who pass along to their offspring a propensity for developing metabolic disturbances, at least in mice. The question as to how experiments with mice bearing the moniker of $B L O B B Y$ can inform us as to the mechanistic origins of how obesity influences offspring health takes an interesting and provocative page out of the developmental history of the female gamete or oocyte (see Wu et al., Mitochondrial dysfunction in oocytes of obese mothers: transmission to offspring and reversal by pharmacological endoplasmic reticulum stress inhibitors, Development (2015) 00, 1-11 doi:10.1242/dev.114850). That the culprits in this scenario had something to do with the health, well-being, and numbers of oocyte mitochondria has long been suspected but why this would be so remained a mystery. This elegant study now brings into focus a stress response pathway that in the end curtails the production of mitochondria that would be inherited from mother through the female germ line. Remarkably, preconceptional treatments of obese mice with drugs known to inhibit the ER stress pathway restored oocyte quality and fetal health to a level matching their lean counterparts.

While there is much to uncover as to the pertinence of these findings relative to Homo sapiens, it is heartening to consider that drugs of this class are being developed and tested for a variety of age-related disorders in humans and, like metformin, may someday appear on the ART menu as a useful adjunct therapy for patients dealing with the negative consequences of obesity on their ability to procreate. In the meantime, a prospective attitude that aims to maintain or rectify the unavoidable damage accrued in our developing gametes seems worthy of further consideration. Withstanding the test of time works for long-held traditions, but not so for our gametes who may need a little help from their somatic friends. 